\title{
Efficiently Harvesting Sun Light for Silicon Solar Cells through Advanced Optical Couplers and A Radial p-n Junction Structure
}

\author{
Hsin-Cheng Lee ${ }^{1}$, Shich-Chuan Wu ${ }^{2}$, Tien-Chung Yang ${ }^{1}$ and Ta-Jen Yen ${ }^{1,2, *}$ \\ 1 Department of Material Science and Engineering, National Tsing Hua University, Hsinchiu, Taiwan; \\ E-Mails: hsincheng.lee@gmail.com (H.C.L.); d9531607@oz.nthu.edu.tw (T.C.Y.) \\ 2 National Nano Device Laboratories, Science-Based Industrial Park, Hsinchu, Taiwan; \\ E-Mail: scwundl@yahoo.com.tw (S.C.W.)
}

* Author to whom correspondence should be addressed; E-Mail: tjyen@mx.nthu.edu.tw; Tel.: +886-3-5742171; Fax: +886-3-5722366.

Received: 29 January 2010; in revised form: 8 March 2010 / Accepted: 12 March 2010 / Published: 20 April 2010

\begin{abstract}
Silicon-based solar cells (SCs) promise to be an alternative energy source mainly due to: (1) a high efficiency-to-cost ratio, (2) the absence of environmentaldegradation issues, and (3) great reliability. Transition from wafer-based to thin-film SC significantly reduces the cost of SCs, including the cost from the material itself and the fabrication process. However, as the thickness of the absorption (or the active) layer decreases, the energy-conversion efficiency drops dramatically. As a consequence, we discuss here three techniques to increase the efficiency of silicon-based SCs: (1) photonic crystal (PC) optical couplers and (2) plasmonic optical couplers to increase efficiency of light absorption in the SCs, and (3) a radial p-n junction structure, decomposing light absorption and diffusion path into two orthogonal directions. The detailed mechanisms and recent research progress regarding these techniques are discussed in this review article.
\end{abstract}

Keywords: solar energy; silicon solar cells; thin-film solar cells; photovoltaics; photonic crystals; surface plasmon; particles plasmon; plasmonic structure; radial p-n junction; silicon nanowires 


\section{Introduction}

The increasing demand for energy and the effects of global warming are two related issues attracting more and more attention from the public. Photovoltaics (PVs), which can turn sunlight - the most abundant renewable and clean energy source - into electricity, provide a great solution to the current lack of sufficient long-term energy supplies. The earth receives solar radiation at levels of up to $\sim 1.7 \times 10^{17} \mathrm{~W}$ in the upper atmosphere whereas the rate of current worldwide energy consumption is $\sim 10,000$ times smaller, at $\sim 1.6 \times 10^{13} \mathrm{~W}[1,2]$. Owing to the eternality of solar energy and the cleanness of solar-energy conversion, photovoltaic technology as an energy source would be a good alternative to fossil energy, once the cost of a SC could be comparable with the cost of fossil fuel (ca. US\$0.05-0.13/kWh) [3].

In 1839, Henri Becquerel first observed the photovoltaic effect [4] (the conversion of light into electricity), which also presents the basic concept behind SCs. Over 100 years later, Daryl Chapin et al. at Bell Labs found that doped crystalline silicon was very sensitive to light and could be a candidate for SCs [5]; shortly thereafter, researchers invented the prototype for practical SCs made from silicon featuring a single $\mathrm{p}-\mathrm{n}$ junction. The silicon-based $\mathrm{p}-\mathrm{n}$ junction SCs have matured gradually owing to improvements in the silicon industry's manufacturing techniques. To date, silicon-based SCs including wafer-based and thin-film technologies have dominated the PV market (more than $90 \%$ thereof) [6], because these SCs meet the following two criteria for the manufacture of a low-cost "clean-energy conversion" system: (1) abundance of raw material, and (2) an absence of negative environmental, health, and safety issues [7]. A report from Yole Development in 2007 even predicted that the dominant photovoltaic technology would be silicon-based cells [8].

In contrast with today's widely used energy sources, both prohibitive expenses and material shortages of wafer-based silicon SCs prevent substantial use of silicon PV worldwide. As a result, those aforementioned issues of wafer-based solar technology have turned industrial players' attention to thin-film modules, which hold the promise of reducing both the cost and the consumption of relevant materials. Thin-film PV promises several particular benefits such as a more economical cost of processing, less reduction in light-induced degradation, significant flexibility of cells, and a decrease in dark current resulting from bulk recombination. As the thickness of the absorption layer decreases to thinner than $10 \mu \mathrm{m}$, however, the energy-conversion efficiency drops dramatically. Therefore, to make low-cost thin-film silicon SCs work more efficiently, such a system would need powerful optical couplers that enhance both light coupling and absorption. It is possible to achieve light-trapping by forming a wavelength-scale texture at the top or bottom of the active layer within wafer-based SCs, and large increases in photocurrent have been achieved in this way [9,10]. However, a rough material surface results in not only an increase in surface recombination but low quality of deposited materials on it. Besides, for thin-film SCs, the thickness of the texture structure is almost on the same scale as that of the active layer. Thus, conventional texture structure is not suitable for thinfilm PV technology, and a new concept or a new structure for enhancing light coupling and absorption of SCs is needed.

In Sections 2 and 3, we briefly review two advanced optical coupling techniques, including PC techniques and plasmonic-enhancing techniques. PC techniques are mainly used as a back reflector of SCs to increase the optical path length inside active layer, hence increasing the efficiency of SCs. 
Plasmonic enhancing techniques are based on surface plasmonic resonances at the interface between metal and dielectrics or particle plasmonic resonances by nanoparticles. Both plasmonic resonances can enhance the intensity of electric field locally, therefore increasing the absorption inside thin-film SCs. The thicknesses of active layer in the thin-film SCs we discuss in these two sections are in the range of 0.1 to $10 \mu \mathrm{m}$. In Section 4, we introduce a new concept of architecture, a radial p-n junction structure, which can decompose the light absorption and diffusion path and can further increase the energy-conversion efficiency of silicon-based SCs.

\section{Photonic Crystal Techniques}

A photonic crystal (PC) is defined as a periodic dielectric structure with refractive index periodicity of the same order as the wavelength of electromagnetic radiation inside. The concept was first described by Yablonovitch and John [11-13] about two decades ago. In general, there are three types of PCs: one-, two-, and three-dimensional PCs (1D, 2D, and 3D PCs). Figure 1 shows the schematic pictures of the three PC types, where the high- and low-index materials as represented in red and blue respectively are arranged in a certain spatial period, $c$.

Figure 1. Schematics of a 1D, 2D and 3D photonic crystal. The colors represent materials with different dielectric indices. The spatial period of the material is called the lattice constant, $c$.

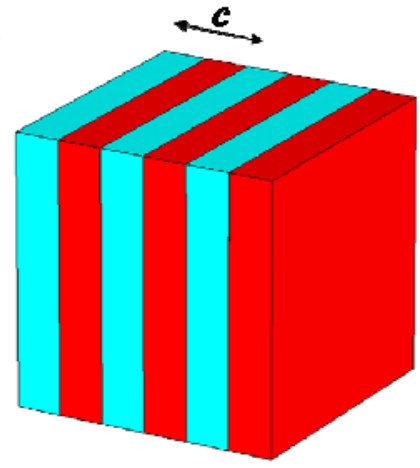

1-D

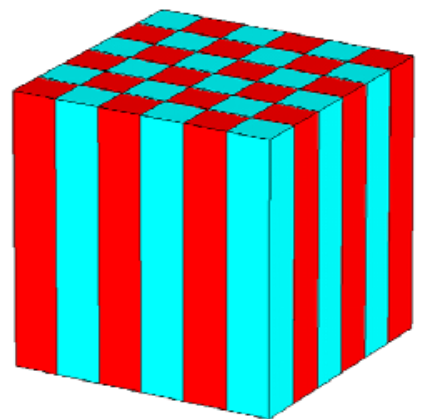

2-D

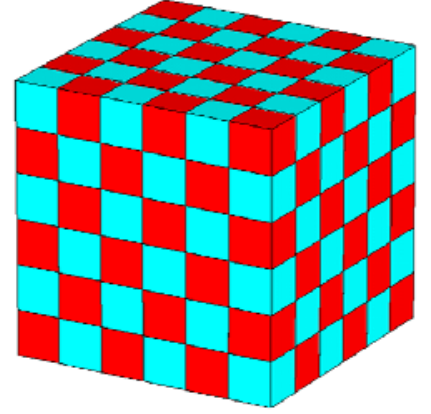

3-D

In a PC, a periodic potential, which originates from alternative changes of the refractive index, results in a band structure for photons. This concept for photons in a PC is analogous to the case for electrons in a crystalline semiconductor. Based on the band structure of a PC, the optical properties including transmittance and reflectance can be easily described. Therefore, we can manipulate light by PCs and exploit such patterned structures to enhance the photon absorption within SCs. There are usually two ways to use the absorption enhancement of SCs: one is to implement a diffraction grating and a rear reflector; the other rests on the sub-wavelength patterning of the absorbing layer itself, which behaves as a planar PC.

A Bragg reflector, which is known as a one-dimensional PC along a light-propagating direction, can function as rear mirrors under the active layer to double the optical path length $[14,15]$. In theory, researchers could enhance path length at more than fifty times the cells' thickness by combining a Bragg reflector with both an ideally roughened front surface and a lossless material of PC [16]. So far, 
the best experimental result for the enhancement of path length is, however, around 10 times the cells' thickness [17]. Some researchers use modulated distributed Bragg reflectors (DBR) to confine more broadband light, as shown in Figure 2 [18]. These researchers proposed a simple modulated PC for extending the region of high reflectance by piling up PCs with different periodicities corresponding to different "band-gaps." The resulting modulated structure becomes a combination of two PC parts in this case - one with smaller periodicity, as shown in Figure 2(a), and the other with greater periodicity, as shown in Figure 2(b). The upper part ensures the high reflectance of short wavelengths of light, while the second part exhibits a high reflectance for longer wavelengths, thereby achieving sufficient broadband reflectance, as shown in Figure 2(c).

Figure 2. Reflectance of PC structures (symbols-measured, lines-simulated) based on a-Si:H and a-SiNx:H layers deposited on a glass substrate. (a) PC_1, (b) PC_2, and (c) modulated PC made of PC_1 and PC_2. The schematics of the PC structures,

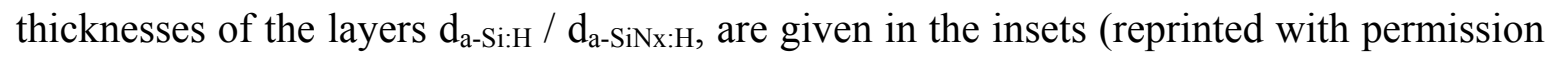
from [18]. (C2009, American Institute of Physics).

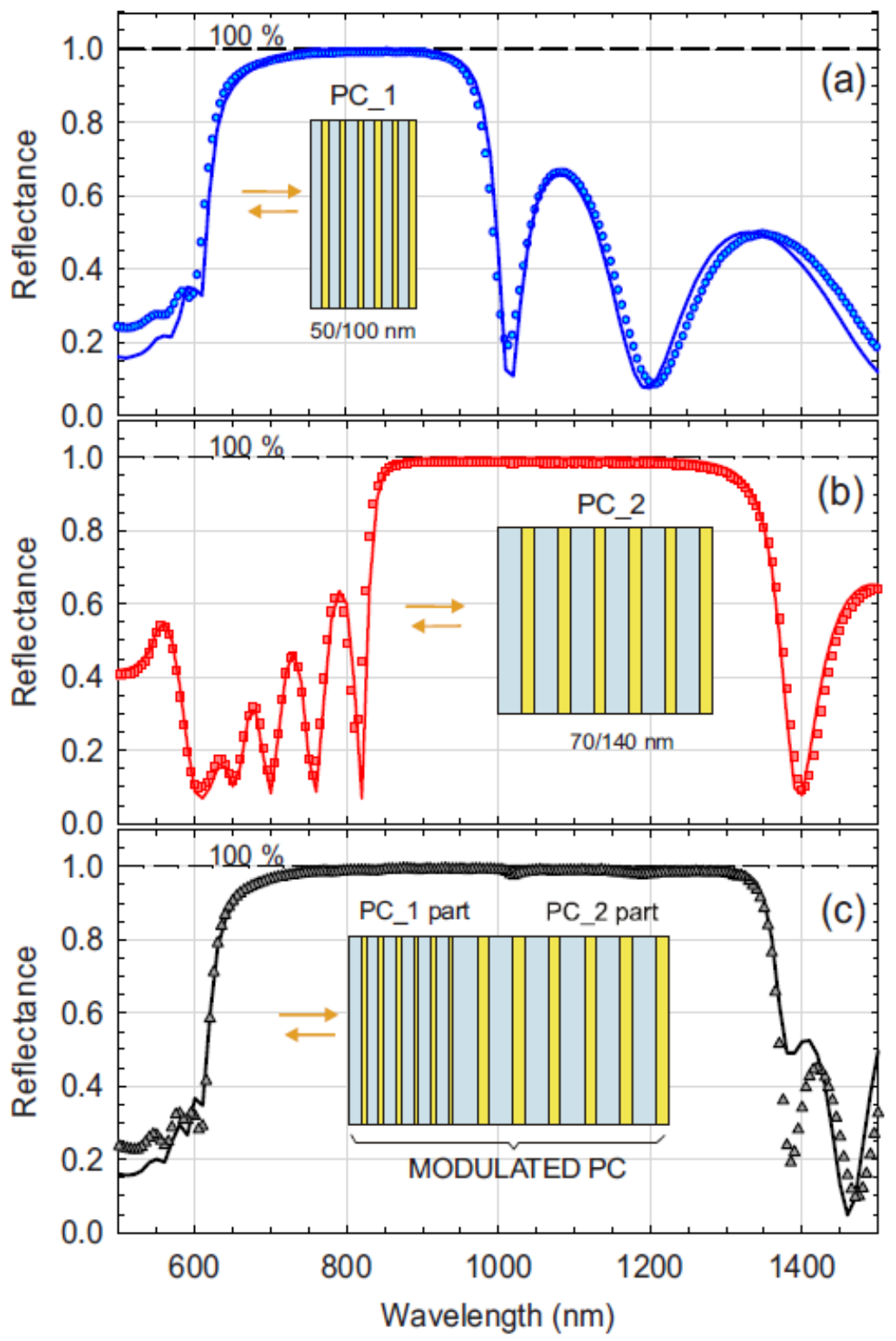


Although the ability of PC back-reflectors to enhance absorption is encouraging, significant challenges arise when attempting to incorporate the light-trapping technique in PV devices. An in-plane one-dimensional PC can function to form large angle diffractions [19-21], thereby increasing the optical path length as well. Yet periodic gratings are not so useful owing to huge transmission losses. The schematics of reference cells without back structure, "DBR-only," and "grating-only" cells are shown in Figure 3(a,b,c), respectively. Zeng et al. [22] demonstrated a potential approach that combines the diffraction gratings with the DBR in the form of textured PC (TPC), shown in Figure 3(d). The short-circuit current enhancement of such a design can reach levels of $28.3 \%$ and $45.2 \%$ for cells with thicknesses of $5 \mu \mathrm{m}$ and $2 \mu \mathrm{m}$, respectively. Some researchers have described the underlying physical mechanisms that give rise to absorption enhancements in thin silicon film featuring PC back-reflectors, and have described hurdles that researchers must surmount in order to reduce-to-practice a PC back-reflector operating as an actual PV device [23, 24].

Figure 3. Schematics of thin-film silicon SCs with different back structures: (a) without any design for reference, (b) DBR only, (c) Grating-only, (d) textured PC (TPC) (reprinted with permission from [22]. (C) 2008, American Institute of Physics).

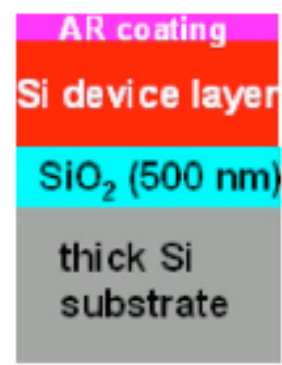

(a) Reference

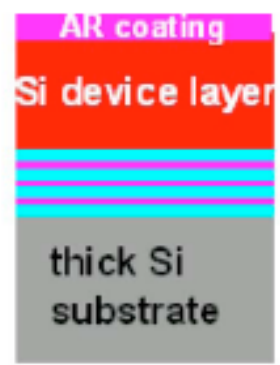

(b) DBR-only

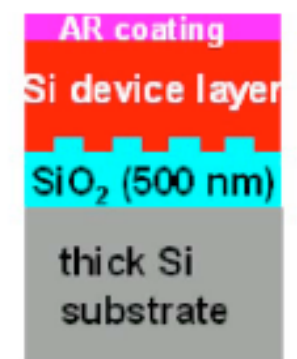

(c) Grating-only

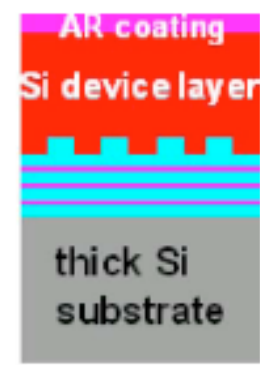

(d) TPC

Alongkarn Chutinan et al. used PCs as an absorbing layer and a good light coupler between air and PCs for the broad solar spectrum as shown in Figure 4. It was demonstrated that there could be both a significant improvement in optical absorption and accordingly attainment of enhanced energy-conversion efficiency [25]. The relative increases of $11.15 \%$ and $3.87 \%$ relative to the conventional design are achieved for $2 \mu \mathrm{m}$ and $10 \mu \mathrm{m}$ thicknesses, respectively.

Figure 4. Schematics for PC SCs showing (a) the entire structure and (b) the front coupler region. (Reused with permission from [25]. (C) 2009, Optical Society of America).

(a)

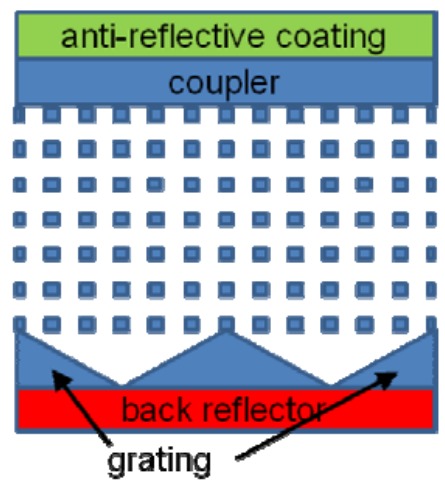

(b)

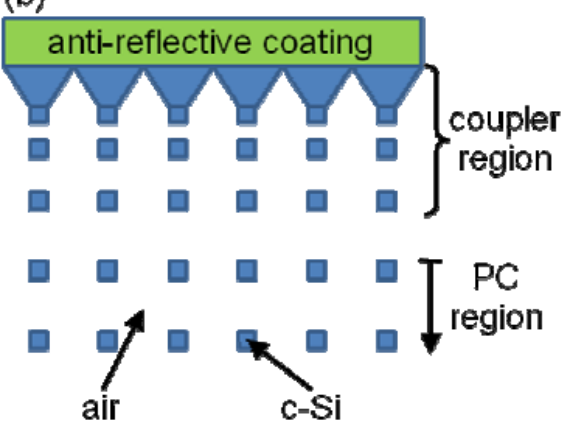


From the above description, we know that the design of PCs would constrain the photons (which are inside the cell layer) through a multi-bouncing process taking place between the front-side and back-side electrodes. Thus, the absorption ratio can be increased because of longer traveling path of incident light inside the active layer. To create such an optical resonant cavity, all the related research focuses on reaching or overcoming the $4 \mathrm{n}^{2}$ enhanced factor (n presents the refractive index of an active layer) [26]. Maximal enhancement (complete absorption) at the resonant frequency in an arbitrarily weakly absorbing material is obtainable by matching the cavity decay time with the intrinsic absorption decay time of the material together with the use of back reflectors. [26]

\section{Plasmonic Enhancing Techniques}

Surface plasmon is a coherent charge-density oscillation existing at the interface between two media with opposite-sign dielectric constants, typically metals and dielectrics [27]. The charge-density wave is associated with an electromagnetic wave, whose electric field reaches the maximum at the interface and decays evanescently within both media. Figure 5 shows a schematic for surface plasmon traveling at the interface between the dielectric layer and the metallic layer. The features of surface plasmon exhibit many special properties, like intense absorption and an enhanced electromagnetic field, which have been achieved in various applications including bio-sensing [28-32] and bio-tagging [33,34], nanoscale optical devices [35], near-field optical microscopy [36], and sub-wavelength photonics [37]. For an SC, the key to applying "surface plasmon enhancing" techniques is to couple the light from a propagating mode to a guided mode along the interface between the active layer, which is a dielectric usually, and the metallic layer. Applying surface plasmon resonance to PVs, therefore, results in an increase in the energy-conversion efficiency mainly due to enhanced optical absorption.

Figure 5. A schematic for surface plasmon resonances traveling along the tangential direction at the interface between a dielectric and a metallic layer. The enhanced electric field reaches its maximum at the interface and decays exponentially from the surface.

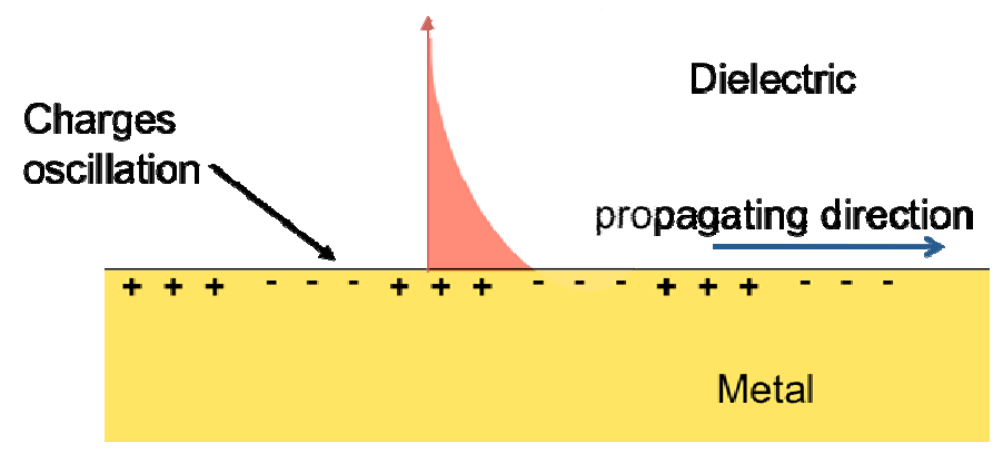

The plasmonic structures are able to enhance the absorption by localizing light at sub-wavelength dimensions and to synergize with the use of ultra-thin-film absorbers, as well as quantum wells, quantum dots, and potentially molecular photovoltaic absorber materials. There are several plasmonic structures used for enhancement of absorption in thin-film SCs, such as nano-scale patterning metallic film and metallic nano-particles distribution. The main purpose to applying those plasmonic structures 
is to increase the traveling path length of photons within an SC active layer. Thus, Polo et al. [38] combined SCs with so-called "nano-engineered metamaterials" [39], i.e., sculptured thin films (STFs) in a dielectric medium, to support multiple plasmonic modes in thin-film SC, thereby, rising the SC's transferring efficiency. Figure 6(a) shows the Kretschmann configuration for such a measurement setup. Their calculations were performed at an interface in the Kretschmann configuration with an aluminum film of thickness $15 \mathrm{~nm}, \mathrm{SNTFs}$ made of $\mathrm{TiO}_{2}$ and the coupling high-index material being ZnSe.

The corresponding incidence-angle-dependent enhanced absorbance at the wavelength of $633 \mathrm{~nm}$ is shown in Figure 6(b). For p-polarized light incidence, there are two sharp peaks of absorption at the angle of incidences of $49.91^{\circ}$ and $75.73^{\circ}$; for s-polarized light incidence, there is one peak of absorption at $55.32^{\circ}$.

Figure 6. (a) Schematic of the Kretschmann configuration. (b) Absorbance as a function of the angle of incidence in the Kretschmann configuration. The solid line is for the absorbance of p-polarized incident light, and the dashed line for the absorbance of s-polarized light. The two sharp peaks in the solid line at $49.91^{\circ}$ and $75.73^{\circ}$ indicate the excitation of surface plasmon wave modes by incident p-polarized light, and the sharp peak in the dashed line at $55.32^{\circ}$ indicates the excitation of an suface plasmon wave by incident s-polarized light (reprinted with permission from the authors of [38]).
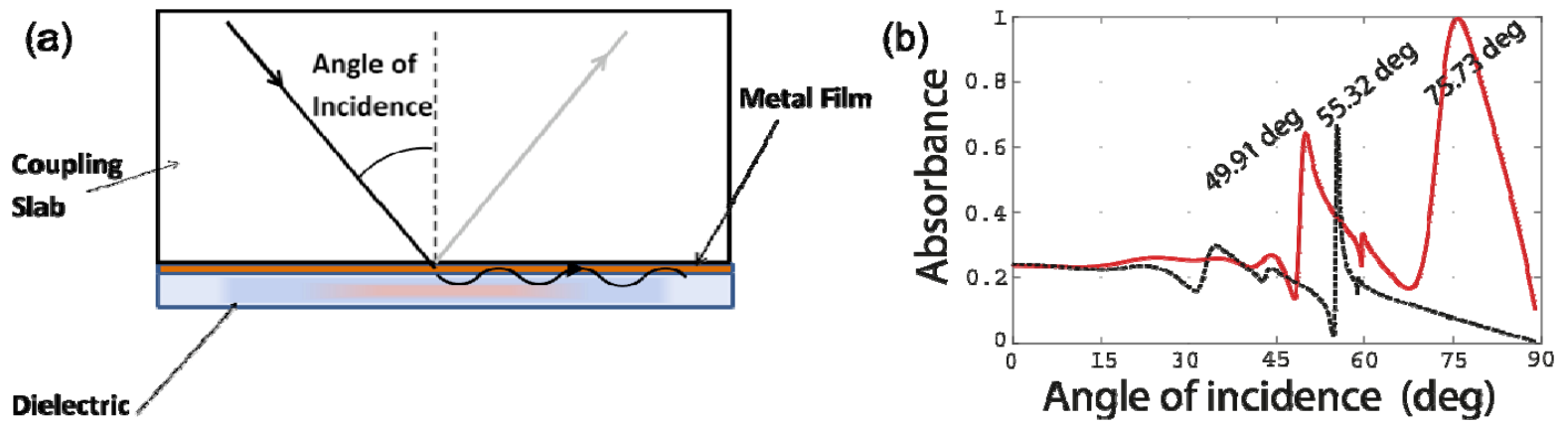

Moreover, Ferry et al. [40] proposed a metallic, subwavelength, and polarization-independent groove structure (shown in Figure 7) under the silicon layer; moreover, the observed enhancement factor relative to a no-groove control set is up to 2.5 at the longest wavelengths $(\sim 1100 \mathrm{~nm})$. At the surface plasmonic resonance frequency, the enhanced local fields near the scatterers (grooves) lead to increased incoupling into the photonic waveguide modes inside the silicon layer, and the surface plasmon is bound to the vicinity of the scattering object because of low group velocity. Figure 8 shows absorption enhancement for a $100 \mathrm{~nm}$ wide by $50 \mathrm{~nm}$ deep Ag groove for different thicknesses of silicon film, relative to the identical thin-film with no groove. For the $200 \mathrm{~nm}$ thick layer of silicon in Figure 7, the incoupling generally increases with increasing wavelength, except at the frequencies near Fabry-Pérot resonances [40]. For the $150 \mathrm{~nm}$ thick film where the film resonance shifts to $580 \mathrm{~nm}$, the structure clearly exhibits enhancement regarding both resonance and the band edge, reaching an enhancement factor of 2.5 at $1100 \mathrm{~nm}$ [40]. In general, Reference 40 has demonstrated the strongest absorption enhancement for optically thin layers. 
Energies 2010, 3 
Figure 7. (a) A three-dimensional scheme of a SC with subwavelength grooves tiled on a back Ag contact. (b) The schematic outline of the device layer in the discussion. The constants here are $\mathrm{t}=200 \mathrm{~nm}$ (thickness of silicon layer), $\mathrm{d}=50 \mathrm{~nm}$ (depth of a $\mathrm{Ag}$ groove), and $\mathrm{w}=100 \mathrm{~nm}$ (width of a $\mathrm{Ag}$ groove) (reprinted with permission from [40]. C) 2008, American Chemical Society).
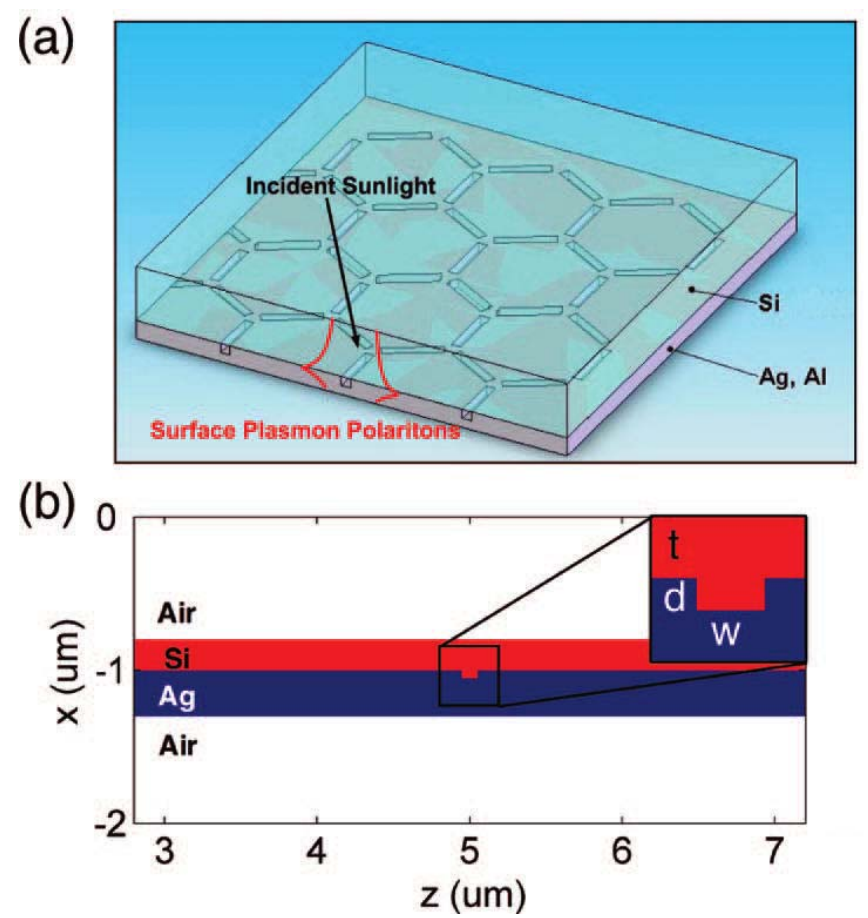

Figure 8. Absorption enhancement for a $100 \mathrm{~nm}$ wide by $50 \mathrm{~nm}$ deep $\mathrm{Ag}$ groove for different thicknesses of silicon film, relative to the identical thin-film with no groove (reprinted with permission from [40]. (C) 2008, American Chemical Society).

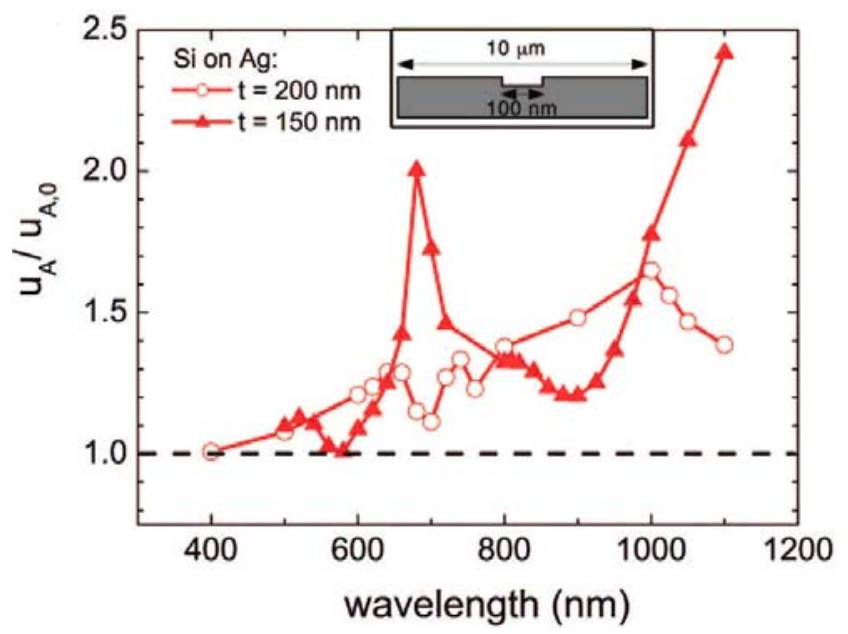

In addition to metal films or metallic nanopatterns, metallic nanoparticles support so-called localized surface plasmon resonances (or nanoparticle plasmon resonances) [41]. Catchpole et al. [42] described the state of the art for particles' plasmonic enhancing techniques. Using their report to conduct pioneering work in the area of plasmonic enhancement of light-sensitive devices, Stuart and 
Hall showed that an enhancement in the photocurrent of a factor of 18 could be achieved for a $165 \mathrm{~nm}$ thick silicon-on-insulator photo-detector at a wavelength of $800 \mathrm{~nm}$ featuring Ag nanoparticles on the surface of the device [43]. Subsequently Schaadt et al. deposited Au nanoparticles on highly doped wafer-based SCs, obtaining enhancements of up to $80 \%$ at wavelengths around $500 \mathrm{~nm}$ [44]. Derkacs et al. used Au nanoparticles on thin-film amorphous silicon SCs to achieve an $8 \%$ overall increase in conversion efficiency [45]. Recently, Pillai et al. deposited Ag particles on $1.25 \mu \mathrm{m}$ thick silicon-on-insulator SCs and planar wafer based cells, and achieved overall photocurrent increases of $33 \%$ and $19 \%$ respectively [46]. Catchpole et al. [47] also demonstrated theoretically that particle shape is a crucial parameter determining the light trapping efficiency: path length enhancements in thin films of up to a factor of 30 were found for optimized shapes. There are two basic mechanisms that currently explain photocurrent enhancement by metal particles incorporated into or on SCs: light scattering and near-field concentration of light. The contribution of each mechanism depends mostly on particle size, the strength of the semiconductor's absorption, and the electrical design of the SCs.

The nanoparticle plasmon resonances can readily be tuned into the ideal spectral range for PV applications by means of size, distribution, shape, and local dielectric environment. The optical cross sections for light absorption and scattering resulting from these nanoparticle plasmons are extremely high and may extinguish most of the incident light within layers, where the effective metal thickness (volume metal per unit area) is only a few nanometers. If the oscillator strength associated with these excitations could efficiently be coupled to useful electron-hole pair production in an SC layer, the thickness of thin-film PV layers $(\sim 1$ to $10 \mu \mathrm{m})$ of present-day could be reduced by a factor up to $10^{2}$ [48] (based on the Thomas-Reiche-Kuhn sum rule [49]). This would pave the way for a new class of SCs, for which materials and concepts not applicable to thicker layers, could be viable.

Catchpole et al. [42] focused on metal-particle scattering as a means of enhancing entrapment of light in thin-film SCs because this type of scattering is the mechanism behind the enhancement in most of the experimental works that have been reported. Figure 9(a) shows a calculated polar plot of the radiation pattern for an electric dipole with a dipole moment parallel to the surface, placed $20 \mathrm{~nm}$ above a silicon substrate (blue dashed line). The radiation pattern for an electric dipole in free space is plotted for reference (black solid line). For the dipole above a silicon surface, only a tiny, barely visible fraction of the light is radiated into the air, while the vast majority (96\%) is radiated into the silicon. The effectiveness of this transfer of energy from a dipole to a high-index substrate has been pointed out by Soller et al. [50], and is related to the high density of optical modes within the silicon. Figure 9(b) shows radiation patterns of dipoles placed at $20 \mathrm{~nm}$ and $60 \mathrm{~nm}$ from the substrate, respectively. At larger distances from the substrate, a reduced fraction of the light is radiated into the substrate

(e.g., $84 \%$ for a dipole $60 \mathrm{~nm}$ from the substrate).

Additionally, Catchpole et al. [47] revealed details regarding the fundamental design principles for increased SC efficiency based on using light trapping and light scattering relative to metal nanoparticles. According to their study, cylindrical and hemispherical particles lead to much higher enhancement of fraction of light scattered into the substrate than spherical particles, shown in Figure 10, because of enhanced near-field coupling. Besides, Ag particles yield much higher path-length enhancements than Au particles. The scattering cross section of the particles is very sensitive to the thickness of a spacer layer at the substrate, which provides additional tunability for 
designing particle arrays. Meanwhile, the aforementioned researchers investigated the relationship between the maximum enhancement of path length and the fraction of light scattered into the substrate, as shown in Figure 11.

Figure 9. (a) Radiation patterns for a point dipole oriented parallel to the surface at a distance of $20 \mathrm{~nm}$ from a Si substrate (blue dashed line). The radiation pattern for the case of free space is shown for reference (black solid line). (b) Radiation patterns for a parallel point dipole $20 \mathrm{~nm}$ (blue dashed line) and $60 \mathrm{~nm}$ (red solid line) from a Si substrate (reused with permission from [42]. (C) 2008, Optical Society of America).

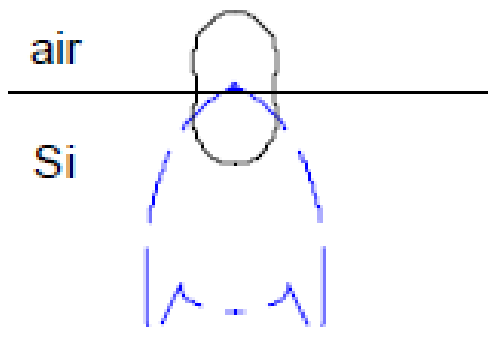

(a)

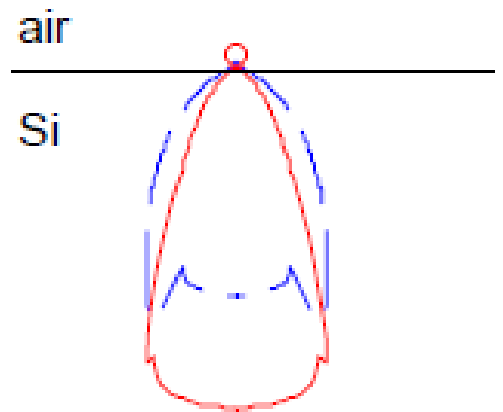

(b)

Figure 10. Fraction of light scattered into the substrate, $f_{\text {subs }}$, for Ag particles on a $10 \mathrm{~nm}$ thick $\mathrm{SiO}_{2}$ underlayer on Si: a cylinder with diameter $d=100 \mathrm{~nm}$ and height $h=50 \mathrm{~nm}$; a $100 \mathrm{~nm}$ diameter hemisphere; a $100 \mathrm{~nm}$ diameter sphere; and a $150 \mathrm{~nm}$ diameter sphere. Also plotted is $f_{\text {subs }}$ for a parallel electric dipole that is $10 \mathrm{~nm}$ from a Si substrate. $\left(f_{\text {subs }}\right.$ is the fraction of light scattered into the substrate) (reprinted with permission from [47]. (C) 2008, American Institute of Physics).

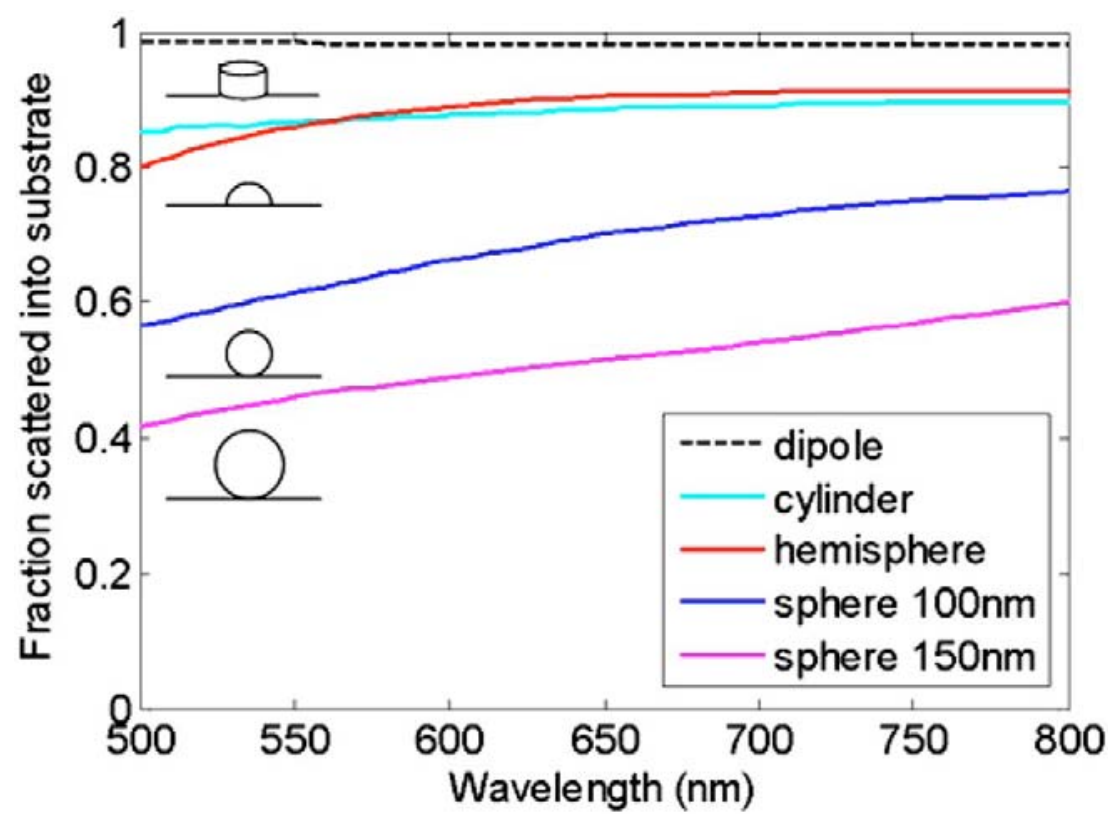


Figure 11. Maximum path length enhancement for the same geometries in Figure 10 at a wavelength of $800 \mathrm{~nm}$. Absorption within the particles is neglected for these calculations, and an ideal rear reflector is assumed. The line is a guide for the eyes. Insets: (top-left) angular distribution of scattered power for a parallel electric dipole that is $10 \mathrm{~nm}$ above a Si surface and Lambertian scatterer; (bottom-right) geometry considered for calculating the path length enhancement (reprinted with permission from [47]. (C) 2008, American Institute of Physics).

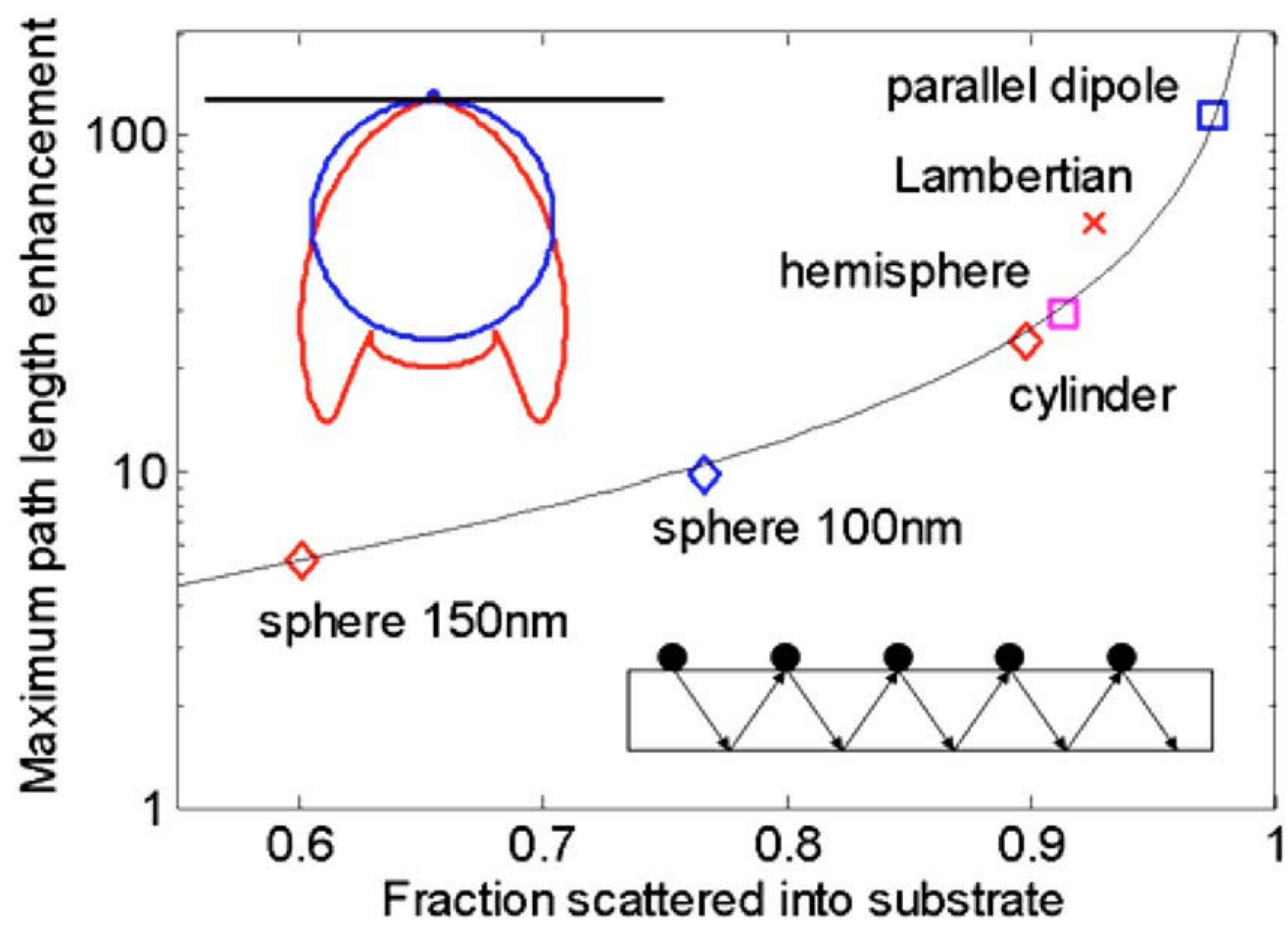

Moreover, Akimov et al. [51,52] studied the plasmonic effect of Ag nanoparticles on the surface of an $a-\mathrm{Si}: \mathrm{H}$ thin-film SC on light trapping inside the photo-active layer. In their calculation experiments, it has been shown that by excitation of higher-order surface plasmon resonances in larger nanoparticles, one can increase the energy transmitted into the amorphous silicon layer, as well as decrease the optical absorption related to the metallic nanoparticles. Thus, the overall broadband optical absorption in the photo-active layer can be significantly improved. Optimizing the size and surface coverage of Ag nanoparticles the maximum enhancement of optical absorption within the $a$ $\mathrm{Si}: \mathrm{H}$ SC can be achieved up to $15.15 \%$, and the corresponding radius and surface coverage of $\mathrm{Ag}$ nanoparticles are 80 $\mathrm{nm}$ and $11 \%$, respectively.

A simple example of metallic nanoparticle enhancement of SC fabrication can be found in the published findings of Derkacs et al. [45]. They fabricated and characterized $a-\mathrm{Si}: \mathrm{H}$ thin-film SCs in which $\mathrm{Au}$ nanoparticles functioned to engineer the transmission and spatial distribution of electromagnetic fields, and Figure 12 presents both the corresponding schematic diagram and an SEM picture of Au nanoparticles. The research team's findings revealed an increase of $8.1 \%$ in short-circuit current density and an increase of $8.3 \%$ in energy-conversion efficiency compared to values achieved in reference devices without Au nanoparticles. 
Figure 12. (a) Schematic diagram of a-Si:H p-i-n SC structure with $\mathrm{Au}$ nanoparticles. (b) SEM image of 100-nm-diameter Au nanoparticles deposited on device surface (reprinted with permission from [45]. C 2006, American Institute of Physics).

(a)

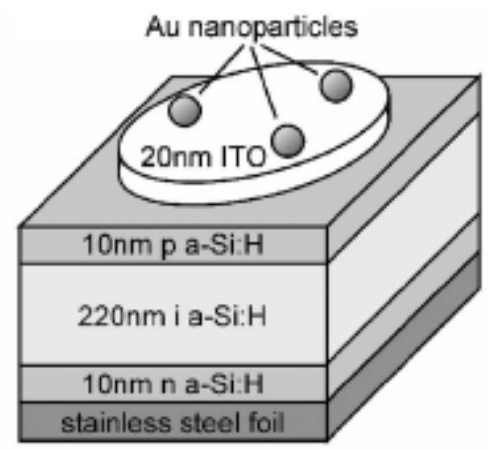

(b)

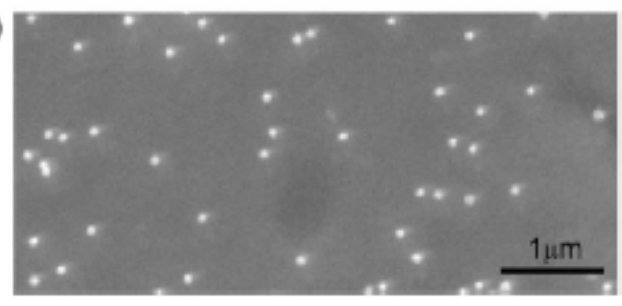

Besides metallic nanoparticles, it has been proven theoretically that dielectric nanoparticles with high permittivity and low dissipation level can significantly enhance photoelectron generation and hence, result in better performance of thin-film SCs [53]. Chen et al. [54] demonstrated the photoresponsitivity and acceptance angle of $\mathrm{n}-\mathrm{ZnO} / \mathrm{p}-\mathrm{Si}$ photodiodes could be enhanced by coating silica nanoparticles on the top surface of photodiodes. Their result provides a potential application in SCs.

\section{A Radial p-n Junction Structure}

Besides optical couplers, researchers have also dedicated their efforts to the device's architecture in order to increase efficiency. In addition to external optical couplers and modified surfaces such as anti-reflection coating, textured surfaces, and fish-bone electrodes, researchers have considered internal structural changes. In 2005, Kayes et al. [55] proposed a radial p-n junction structure model, shown in Figure 13, to decompose the light absorption and diffusion path into two orthogonal directions, and the corresponding conversion efficiency can, in theory, undergo up to a $20 \%$ improvement, which is comparable with bulk crystalline silicon SC. Unlike the conventional planar $\mathrm{SCs}$, the radial $\mathrm{p}-\mathrm{n}$ junction structure can enhance light absorption by increasing the length of the axial direction without increasing the diffusion length along the radial direction. Furthermore, a shorter diffusion path could decrease the electronic recombination caused by impurities. That potential outcome indicates that the demand for high-quality silicon wafers is no longer a requirement for $\mathrm{SC}$, and the removal of this prior requirement makes it possible to reduce costs related to both the purification and the consumption of materials. 
Figure 13. A scheme of a radial p-n junction structure. This structure allows the light absorption (axial direction) to be perpendicular to diffusion path (radial direction).

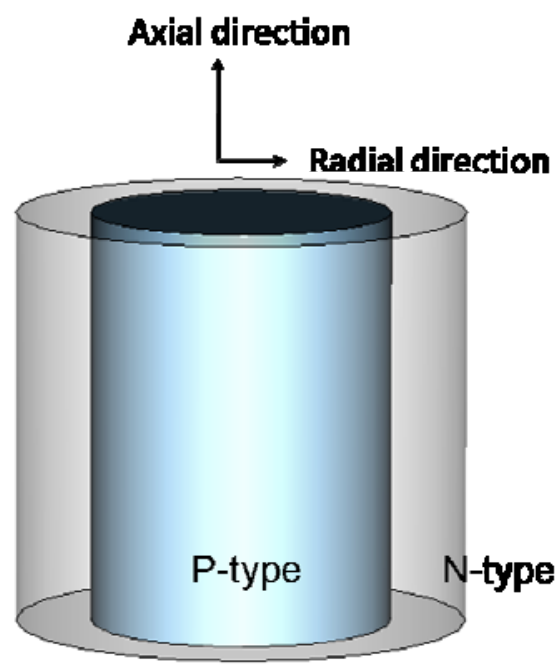

Recent reports that a combination of nanostructured materials and a photovoltaic device could significantly and simultaneously reduce costs and promote efficiency [55-62]. Unique physical properties appear within such small dimensions owing to quantum confinement or high surface energy, which make nanostructured silicon more feasible for photovoltaic devices. One-dimensional nanostructures such as nanotips (NTs), nanorods, and nanowires (NWs) could present great anti-reflection properties [63-66]. The reflection of such structures can be significantly reduced down to $5 \%[64,65]$ or even $1 \%$ [63] for a broadband spectrum from UV to near-IR which matches the solar spectrum.

By combining the aforementioned advantages of a radial $p-n$ junction structure and a one-dimensional structure, there have been proposals and experimental demonstrations regarding SiNWs SCs. Recently, Tsakalakos et al. [60] realized a radial p-n junction SiNWs SC fabricated by a vapor-solid-liquid (VLS) mechanism, following an chemical vapor deposition (CVD) to coat an external shell, as shown in Figure 14(a) and (b). From their report, the NW cells showed spectrally broad external quantum efficiency (EQE), and the peak measurement of EQE was 12\% at $690 \mathrm{~nm}$, as shown in Figure 14(c). However, the large-area SiNWs cannot be obtained by the VLS mechanism easily.

An alternative approach to achieving large-area crystalline SiNWs is to use room-temperature aqueous electroless etching [61] and a doped thin-film silicon was deposited as a shell to form p-n core-shell SiNW SCs. In general, the typical wire diameter including the shell is $350-400 \mathrm{~nm}$, which gives a shell thickness of about $150 \mathrm{~nm}$ on top of the 50-100 nm initial NW core, as shown in Figure 15. Yet, in opposition to expectations, the overall efficiency of this device was only $0.46 \%$, limited primarily by interfacial recombination and high series resistance. The authors claimed that surface passivation and contact optimization would be critical to future improvements in device performance [61]. 
Figure 14. (a) A schematic cross-sectional view of the SiNWs SC architecture. (b) An SEM plan view of a typical SiNW solar with insets showing a cross-sectional view of the device and a higher magnification of an individual SiNW coated with a-Si and ITO. (c) Measured external quantum efficiency (EQE) for a SiNW cell showing a broad EQE response (reprinted with permission from [60]. (C) 2007, American Institute of Physics).
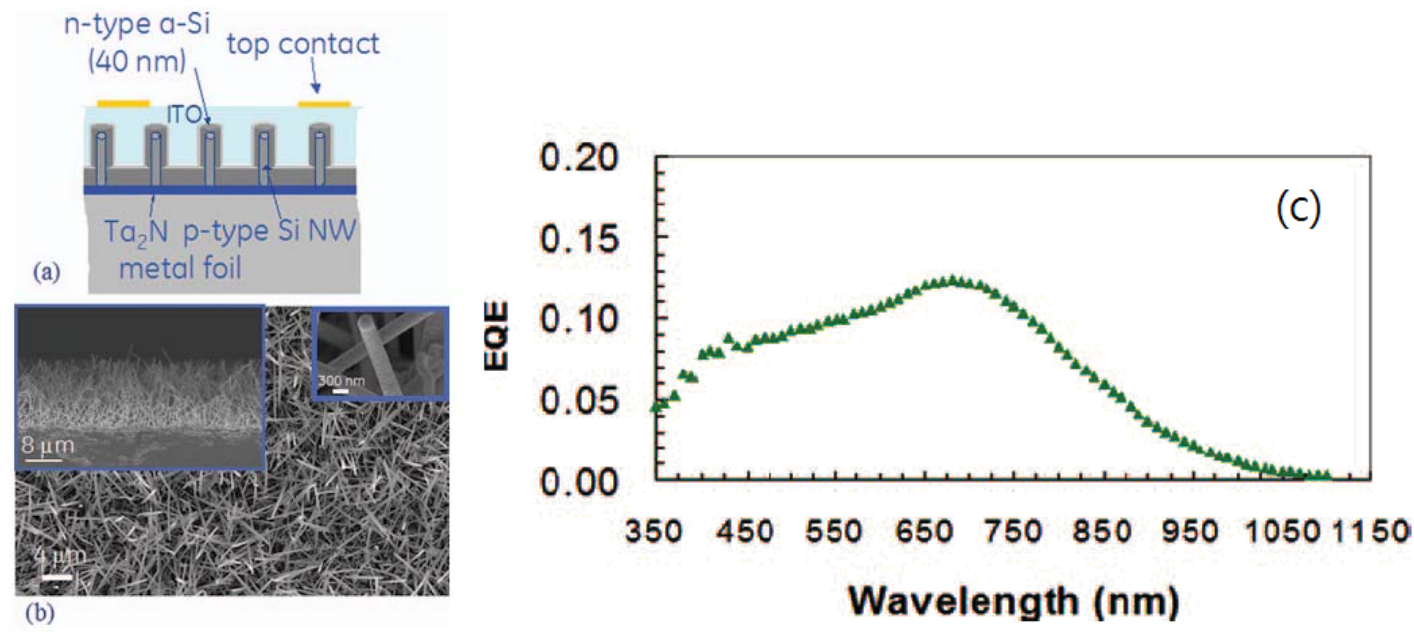

Figure 15. (a) A schematic cell designed with the single crystalline n-Si NW core in brown, the polycrystalline p-Si shell in blue, and the back contact in black. (b) A crosssectional SEM of a completed device demonstrating excellent vertical alignment and dense wire packing. (c) A TEM image showing the single crystalline $\mathrm{n}-\mathrm{Si}$ core and polycrystalline $\mathrm{p}$-Si shell. The inset is the selected area electron diffraction pattern. (d) A TEM image from the edge of the core-shell NW showing nanocrystalline domains (reprinted with permission from [61]. (C) 2008 American Chemical Society).

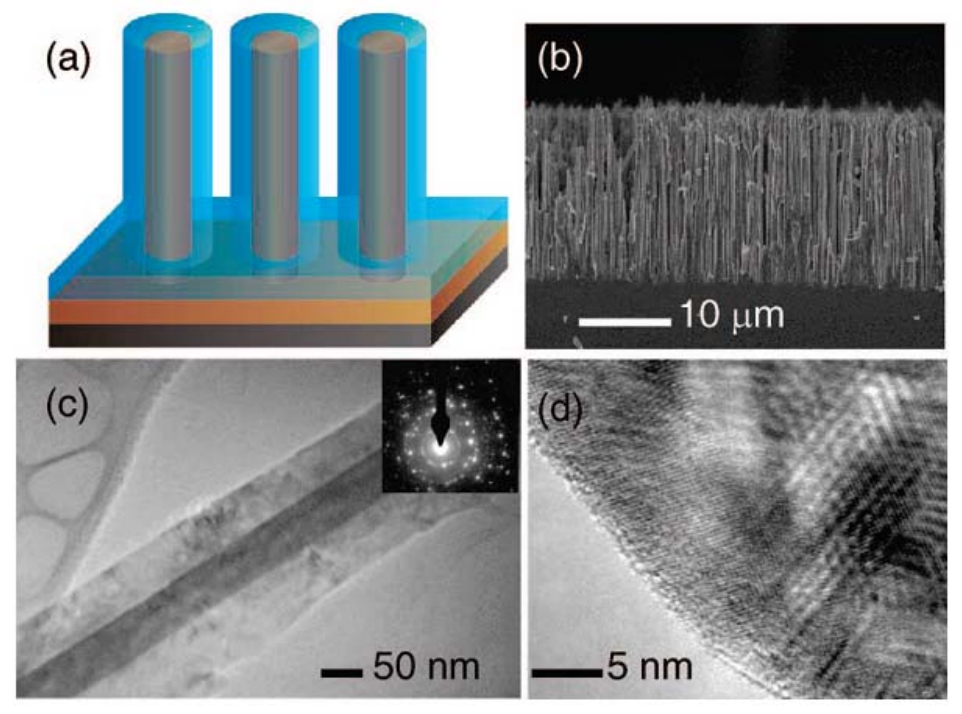

The large area of SiNW SCs provides inherent light-trapping properties based on two potential theoretical frameworks: (1) antenna theory and (2) Mie scattering theory [67]. The light-trapping mechanism makes absorption more efficient without additional antireflection layers. In addition, 
Lieber's group proposed novel designs for NW SCs that would improve energy-conversion efficiency and that include coaxial tandem and multiple quantum-well (MQW) structures, shown in Figure 16 [68]. A radial p-n junction structure relative to SCs provides a potential solution of the next generation silicon SCs. However, this promising device still has many challenges to overcome, such as enormous surface defects and non-uniformity of the shell coating, which can decrease the energy-conversion efficiency of SCs.

Figure 16. New NW structures for improving energy conversion efficiency. (a) Schematics of axial and coaxial NW tandem SCs. (b) Schematic of an MQW NW SC. Pink and blue regions denote p-core and n-shell, respectively; yellow and orange regions are barriers and quantum wells in the i-shell, respectively. (c) Band structure and carrier transport schematics of an MQW NW SC (reproduced from [68] by permission of The Royal Society of Chemistry).

a)

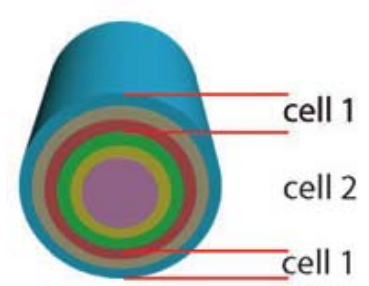

b)

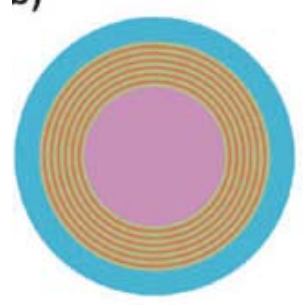

c)

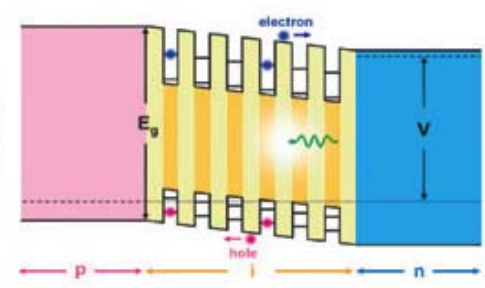

\section{Conclusions}

In this review, we have commented on recent progress in enhancing various techniques that support low-cost silicon-based PVs and that include PCs, plasmonic-enhancing techniques, and a radial p-n junction for SiNW SCs. By placing a PC structure inside a silicon solar cell or by using such a structure as back reflectors, there can be significant increases in absorption efficiency. A plasmonic resonance by either metallic grooves or nanoparticles can also enhance the absorption of SC. The radial $p-n$ junction structure can increase EQE and absorption efficiency as well; anti-reflection properties of such a structure can also facilitate the overall efficiency.

\section{Acknowledgements}

The authors would like to gratefully acknowledge the financial support from National Science Council (NSC 98-2112-M-007 -002 MY3) and Ministry of Economic Affairs (97-EC-17-A- 08-S1-03). 


\section{References}

1. Trenberth, K.E.; Fasullo, J.T.; Kiehl, J. Earth's global energy budget. Am. Meteor. Soc. 2009, 90, 311-323.

2. Energy Information Administration (EIA), International Energy Annual 2006. Available online: http://www.eia.doe.gov/ (accessed on 20 March 2010).

3. Slaoui, A.; Collins, R.R. Advanced inorganic materials for photovoltaics. MRS Bull. 2007, 32, 211-218.

4. Williams, R. Becquerel photovoltaic effect in binary compounds. J. Chem. Phys. 1960, 32, 1505-1514.

5. Chapin, D.M.; Fuller, C.S.; Pearson, G.L. A new silicon p-n junction photocell for converting solar radiation into electrical power. J. Appl. Phys. 1954, 25, 676-677.

6. Van Sark, W.G.J.H.M.; Brandsen, G.W.; Fleuster, M.; Hekkert, M.P. Analysis of the silicon market: Will thin films profit? Energy Policy 2007, 35, 3121-3125.

7. Singh, R. Why silicon is and will remain the dominant photovoltaic material. J. Nanophotonics 2009, 3, 032503-11.

8. Issues and opportunities for the PV industry. Available online: http://www.i-micronews.com/ analysis/Issues-opportunities-PV-industry,401.html (accessed on 20 March 2010).

9. Muller, J.; Rech, B.; Springer, J.; Vanecek, M. TCO and light trapping in silicon thin film solar cells. Solar Energy 2004, 77, 917-930.

10. Meier, J.; Dubail, S.; Golay, S.; Kroll, U.; Faÿ, S.; Vallat-Sauvain, E.; Feitknecht, L.; Dubail, J.; Shah, A. Microcrystalline silicon and the impact on micromorph tandem solar cells. Solar Energy Mater. Solar Cell 2002, 74, 457-467.

11. Yablonovitch, E. Inhibited spontaneous emission in solid-state physics and electronics. Phys. Rev. Lett. 1987, 58, 2059.

12. John, S. Strong localization of photons in certain disordered dielectric superlattices. Phys. Rev. Lett. 1987, 58, 2486.

13. Joannopoulos, J.D.; Johnson, S.G.; Winn, J.N.; Meade, R.D. Photonic Crystals: Molding the Flow of Light; Princeton University Press: Princeton, NJ, USA, 1995.

14. Johnson, D.C.; Ballard, I.; Barnham, K.W.J.; Bishnell, D.B.; Connolly, J.P.; Lynch, M.C.; Tibbits, T.N.D.; Ekins-Daukes, N.J.; Mazzer, M.; Airey, R.; Hill, G.; Roberts, J.S. Advances in Bragg stack quantum well solar cells. Solar Energy Mater. Solar Cell 2005, 87, 169-179.

15. Kuzma-Filipek, I.; Duerinckx, F.; Nieuwenhuysen, K.V.; Beaucarne, G.; Poortmans, J.; Mertens, R. Porous silicon as an internal reflector in thin epitaxial solar cells. Phys. Status Solid A 2007, 204, 1340-1345.

16. Yablonovitch, E.; Cody, G.D. Intensity enhancement in textured optical sheets for solar-cells. IEEE Trans. Electron. Device 1982, 29, 300-305.

17. Nelson, J. The physics of solar cells. In The Physics of Solar Cells; Imperial College: London, UK, 2003; pp. 279-282.

18. Krc, J.; Zeman, M.; Luxembourg, S.L.; Topic, M. Modulated photonic-crystal structures as broadband back reflectors in thin-film solar cells. Appl. Phys. Lett. 2009, 94, 153501-3. 
19. Heine, C.; Morf, R.H. Submicrometer gratings for solar energy applications. Appl. Opt. 1995, 34, 2476-2482.

20. Llopis, F.; Tobİas, I. The role of rear surface in thin silicon solar cells. Solar Energy Mater. Solar Cell 2005, 87, 481-492.

21. Stiebig, H.; Senoussaoui, N.; Zahren, C.; Haase, C.; M,ller, J. Silicon thin-film solar cells with rectangular-shaped grating couplers. Pro. Photovolt.: Res. Appl. 2006, 14, 13-24.

22. Zeng, L.; Bermel, P.; Yi, Y.; Alamariu, B.A.; Broderick, K.A.; Liu, J.; Hong, C.; Duan, X.; Joannopoulos, J.; Kimerling, L.C. Demonstration of enhanced absorption in thin film Si solar cells with textured photonic crystal back reflector. Appl. Phys. Lett. 2008, 93, 221105-3.

23. Bermel, P.; Luo, C.; Zeng, L.; Kimerling, L.C.; Joannopoulos, J.D. Improving thin-film crystalline silicon solar cell efficiencies with photonic crystals. Opt. Express 2007, 15, 1698617000 .

24. O'Brien, P.G.; Kherani, N.P.; Chutinan, A.; Ozin, G.A.; John, S.; Zukotynski, S. Silicon photovoltaics using conducting photonic crystal back-reflectors. Adv. Mater. 2008, 20, 15771582.

25. Chutinan, A.; Kherani, N.P.; Zukotynski, S. High-efficiency photonic crystal solar cellarchitecture. Opt. Express 2009, 17, 8871-8878.

26. Chutinan, A.; John, S. Light trapping and absorption optimization in certain thin-film photonic crystal architectures. Phys. Rev. A 2008, 78, 023825-15.

27. Homola, J.; Yee, S.S.; Gauglitz, G. Surface plasmon resonance sensors: review. Sensor Actuat. B: Chem. 1999, 54, 3-15.

28. Barnes, W.L.; Dereux, A.; Ebbesen, T.W. Surface plasmon subwavelength optics. Nature 2003, 424, 824-830.

29. Moskovits, M. Surface-enhanced spectroscopy. Rev. Modern Phys. 1985, 57, 783.

30. Kneipp, K.; Wang, Y.; Kneipp, H.; Perelman, L.T.; Itzkan, I.; Dasari, R.R.; Feld, M.S. Single molecule detection using surface-enhanced Raman scattering (SERS). Phys. Rev. Lett. 1997, $78,1667$.

31. Nie, S.; Emory, S.R. Probing single molecules and single nanoparticles by surface-enhanced Raman scattering. Science 1997, 275, 1102-1106.

32. Hoa, X.D.; Kirk, A.G.; Tabrizian, M. Towards integrated and sensitive surface plasmon resonance biosensors: A review of recent progress. Biosens. Bioelectr. 2007, 23, 151-160.

33. Schultz, S.; Smith, D.R.; Mock, J.J.; Schultz, D.A. Single-target molecule detection with nonbleaching multicolor optical immunolabels. Proc. Nat. Acad. Sci. 2000, 97, 996-1001.

34. Taton, T.A.; Mirkin, C.A.; Letsinger, R.L. Scanometric DNA array detection with nanoparticle probes. Science 2000, 289, 1757-1760.

35. Maier, S.A.; Brongersma, M.L.; Kik, P.G.; Meltzer, S.; Requicha, A.A.G.; Atwater, H.A. Plasmonics-A route to nanoscale optical devices. Adv. Mater. 2001, 13, 1501-1505.

36. Kalkbrenner, T.; Ramstein, M.; Mlynek, J.; Sandoghdar, V. A single gold particle as a probe for apertureless scanning near-field optical microscopy. J. Microscopy 2001, 202, 72-76.

37. Ditlbacher, H.; Krenn, J.R.; Schider, G.; Leitner, A.; Aussenegg, F.R. Two-dimensional optics with surface plasmon polaritons. Appl. Phys. Lett. 2002, 81, 1762-1764. 
38. Polo, J.; Motyka, M.; Lakhtakia, A. Multiple plasmonic modes with sculptured thin films. SPIE newsroom 2008, doi: 10.1117/2.1200810.1310.

39. Messier, A.L.R. Sculptured Thin Films: Nanoengineered Morphology and Optics; SPIE Press: Bellingham, WA, USA, 2005.

40. Ferry, V.E.; Sweatlock, L.A.; Pacifici, D.; Atwater, H.A. Plasmonic nanostructure design for efficient light coupling into solar cells. Nano Lett. 2008, 8, 4391-4397.

41. Kreibig, U.; Vollmer, M. Optical properties of metal clusters. In Springer Series in Materials Science; Springer: New York, NY, USA, 1995; Volume 25.

42. Catchpole, K.R.; Polman, A. Plasmonic solar cells. Optics Express 2008, 16, 21793-21800.

43. Stuart, H.R.; Hall, D.G. Island size effects in nanoparticle-enhanced photodetectors. Appl. Phys. Lett.1998, 73, 3815-3817.

44. Schaadt, D.M.; Feng, B.; Yu, E.T. Enhanced semiconductor optical absorption via surface plasmon excitation in metal nanoparticles. Appl. Phys. Lett. 2005, 86, 063106-3.

45. Derkacs, D.; Lim, S.H.; Matheu, P.; Mar, W.; Yu, E.T. Improved performance of amorphous silicon solar cells via scattering from surface plasmon polaritons in nearby metallic nanoparticles. Appl. Phys. Lett. 2006, 89, 093103-3.

46. Pillai, S.; Catchpole, K.R.; Trupke, T.; Green, M.A. Surface plasmon enhanced silicon solar cells. J. Appl. Phys. 2007, 101, 093105-8.

47. Catchpole, K.R.; Polman, A. Design principles for particle plasmon enhanced solar cells. Appl. Phys. Lett. 2008, 93, 191113-3.

48. Hägglund, C. Nanoparticle Plasmon Influence on the Charge Carrier Generation in Solar Cells; Doctor Thesis, Chalmers University of Technology: Göteborg, Sweden, 2008.

49. Sakurai, J.J. Modern Quantum Mechanics (Revised Edition); Addison-Wesley: Reading, MA, USA, 1994.

50. Soller, B.J.; Stuart, H.R.; Hall, D.G. Energy transfer at optical frequencies to silicon-on-insulator structures. Optic Lett. 2001, 26, 1421-1423.

51. Akimov, Y.A.; Koh, W.S.; Ostrikov, K. Enhancement of optical absorption in thin-film solar cells through the excitation of higher-order nanoparticle plasmon modes. Optic Express 2009, 17, 10195-10205.

52. Akimov, Y.A.; Ostrikov, K.; Li, E. Surface plasmon enhancement of optical absorption in thin-film silicon solar cells. Plasmonics 2009, 4, 107-113.

53. Akimov, Y.A.; Koh, W.S.; Sian, S.Y.; Ren, S. Nanoparticle-enhanced thin film solar cells: Metallic or dielectric nanoparticles? Appl. Phys. Lett. 2010, 96, 073111-3.

54. Chen, C.P.; Lin, P.H.; Chen, L.Y.; Ke, M.Y.; Cheng, Y.W.; Huang, J.J. Nanoparticle-coated $\mathrm{n}-\mathrm{ZnO} / \mathrm{p}$-Si photodiodes with improved photoresponsivities and acceptance angles for potential solar cell applications. Nanotechnology 2009, 20, 245204.

55. Kayes, B.M.; Atwater, H.A.; Lewis, N.S. Comparison of the device physics principles of planar and radial p-n junction nanorod solar cells. J. Appl. Phys. 2005, 97, 114302.

56. Lewis, N.S. Toward cost-effective solar energy use. Science 2007, 315, 798-801.

57. Gratzel, M. Photoelectrochemical cells. Nature 2001, 414, 338-344.

58. Huynh, W.U.; Dittmer, J.J.; Alivisatos, A.P. Hybrid nanorod-polymer solar cells. Science 2002, 295, 2425-2427. 
59. Tian, B.Z.; Zheng, X.L.; Kempa, T.J.; Fang, Y.; Yu, N.F.; Yu, G.H.; Huang, J.L.; Lieber, C.M. Coaxial silicon nanowires as solar cells and nanoelectronic power sources. Nature 2007, 449, 885-888.

60. Tsakalakos, L.; Balch, J.; Fronheiser, J.; Korevaar, B.A.; Sulima, O.; Rand, J. Silicon nanowire solar cells. Appl. Phys. Lett. 2007, 91, 3.

61. Garnett, E.C.; Yang, P.D. Silicon nanowire radial p-n junction solar cells. J. Amer. Chem. Soc. 2008, 130, 9224-5.

62. Kempa, T.J.; Tian, B.Z.; Kim, D.R.; Hu, J.S.; Zheng, X.L.; Lieber, C.M. Single and tandem axial p-i-n nanowire photovoltaic devices. Nano Lett. 2008, 8, 3456-3460.

63. Huang, Y.F.; Chattopadhyay, S.; Jen, Y.J.; Peng, C.Y.; Liu, T.A.; Hsu, Y.K.; Pan, C.L.; Lo, H.C.; Hsu, C.H.; Chang, Y.H.; Lee, C.S.; Chen, K.H.; Chen, L.C. Improved broadband and quasi-omnidirectional anti-reflection properties with biomimetic silicon nanostructures. Nature Nanotechnol. 2007, 2, 770-774.

64. Peng, K.Q.; Xu, Y.; Wu, Y.; Yan, Y.J.; Lee, S.T.; Zhu, J. Aligned single-crystalline Si nanowire arrays for photovoltaic applications. Small 2005, 1, 1062-1067.

65. Peng, K.Q.; Wu, Y.; Fang, H.; Zhong, X.; Xu, Y.; Zhu, J. Uniform, axial-orientation alignment of one-dimensional single-crystal silicon nanostructure arrays. Angew. Chemie Int. Ed. 2005, 44, 2737-2742.

66. Chen, Q.; Hubbard, G.; Shields, P.A.; Liu, C.; Allsopp, D.W.E.; Wang, W.N.; Abbott, S. Broadband moth-eye antireflection coatings fabricated by low-cost nanoimprinting. Appl. Phys. Lett. 2009, 94, 263118-3.

67. Tsakalakos, L.; Balch, J.; Fronheiser, J.; Shih, M.Y.; LeBoeuf, S.F.; Pietrzykowski, M.; Codella, P.J.; Korevaar, B.A.; Sulima, O.; Rand, J.; Davuluru, A.; Rapol, U. Strong broadband optical absorption in silicon nanowire films. J. Nanophoton. 2007, 1, 10.

68. Tian, B.; Kempa, T.J.; Lieber, C.M. Single nanowire photovoltaics. Chem. Soc. Rev. 2009, 38, 16-24, doi: 10.1039/b718703n.

(C) 2010 by the authors; licensee MDPI, Basel, Switzerland. This article is an open-access article distributed under the terms and conditions of the Creative Commons Attribution license (http://creativecommons.org/licenses/by/3.0/). 